\title{
Reconsidering the origin of the X-ray emission lines in GRB 011211
}

\author{
F. Tavecchio ${ }^{1}$, G. Ghisellini ${ }^{1}$, and D. Lazzati ${ }^{2}$ \\ 1 INAF-Osserv. Astron. di Brera, via Bianchi 46, 23807 Merate, Italy \\ 2 Institute of Astronomy, University of Cambridge, Madingley Road, CB3 OHA Cambridge, UK
}

Received 14 May 2003 / Accepted 29 September 2003

\begin{abstract}
We reanalyze the XMM-Newton data of GRB 011211 showing that the spectral features, interpreted by Reeves et al. $(2002,2003)$ as due to thermal emission from a collisionally ionized plasma, can be also reproduced by a reflection model (with ionization parameter $\xi \sim 10^{2}$ ). We discuss the implications of this interpretation, estimating the total mass required in the simplified case of a funnel geometry. We conclude that a moderate clumping of the reprocessing material (corresponding to a filling factor of the order of $f \sim 10^{-3}$ ) is required. Finally we show that, if this interpretation is correct, a bright quasi-thermal component is expected in the optical-UV band (containing about $90 \%$ of the luminosity of the illuminating continuum), whose presence can be used to test the reflection model.
\end{abstract}

Key words. gamma rays: bursts - line: formation - radiation mechanisms: general

\section{Introduction}

The study of spectral features (absorption, emission lines) in the X-ray spectra of afterglows of Gamma-Ray Bursts is thought to be one of the most powerful tools to probe the circumburst environment. Even if the direct observation of the central engine is not possible, it might be possible to get indirect indication of the composition and structure of the GRB environment using emission lines (for recent reviews see Lazzati 2002a and Böttcher 2002). Until now, emission features have been detected (in some cases with only marginal significance) in 7 GRBs, namely GRB 970508 (Piro et al. 1999), GRB 970828 (Yoshida et al. 2000), GRB 991216 (Piro et al. 2000), GRB 000214 (Antonelli et al. 2000), GRB 011211 (Reeves et al. 2002, 2003), GRB 020813 (Butler et al. 2003) and GRB 030227 (Watson et al. 2003). In most of these cases the X-ray spectrum shows evidence of the iron line, largely interpreted as due to recombination, either in a photoionized (e.g. Vietri et al. 2001) or in a collisionally-ionized (thermal) plasma (e.g. Paerels et al. 2000).

Recently, Reeves et al. $(2002,2003)$ used a thermal model to fit the X-ray spectrum of the afterglow of GRB $011211 \mathrm{ob}-$ served by XMM-Newton (unfortunately the observation is affected by pointing problems and the real significance of the lines has been criticized on different grounds by Borozdin \& Trudolyubov 2002 and Rutledge \& Sako 2003). The residuals of a fit to the data with an absorbed power-law model only show evident features located at $0.7,0.9$ and $1.2 \mathrm{keV}$.

Send offprint requests to: F. Tavecchio, e-mail: tavecchio@merate.mi.astro.it
Reeves et al. show that a good fit to the data can be obtained assuming a thermal plasma model $(k T \sim 4 \mathrm{keV})$ enriched in light metals $(\mathrm{Ca}, \mathrm{Mg}, \mathrm{Si}, \mathrm{S}, \mathrm{Ar})$. The viability of such a model is critically discussed by Lazzati (2003) who concludes that a thermal origin of the emission puts severe constraints on the parameters of the emission region: in particular the emitting gas must be strongly clumped, with volume filling factors of the order of $\sim 10^{-6}-10^{-7}$. Another problem comes from the fact that, in order to fit the spectrum with the thermal VMEKAL model, Reeves et al. are forced to assume overabundance of $\mathrm{Mg}, \mathrm{S}, \mathrm{Si}$, $\mathrm{Ar}, \mathrm{Ca}$ (about a factor of 10 larger than the solar abundance), but a solar abundance of iron, clearly not easily explainable in a standard hypernova scenario (Woosley 1993), since the exploding star is thought to produce a large amount of $\mathrm{Ni}, \mathrm{Co}$ and $\mathrm{Fe}$ (e.g. Woosley \& Weaver 1995).

Reeves et al. (2003) also considered the possibility that the spectrum is produced through the reflection by an ionized slab (Ross \& Fabian 1993; Ballantyne \& Ramirez-Ruiz 2001), but concluded that this model (either "pure" or with an underlying power-law) cannot reproduce the data. However (as explicitly noted by the authors), most of the discrepancy between the model and the data is localized in the region around $1 \mathrm{keV}$, where lines from elements not included in the ionized reflection model, such as S, Ar and $\mathrm{Ca}$, are expected.

More recently, Butler et al. (2003) and Watson et al. (2003) reported the possible detection of emission lines of light elements (but not Iron) in the Chandra HETGS spectrum of GRB 020813 and in the XMM-Newton spectrum of the afterglow of GRB 030227. These features can be identified as lines of hydrogen-like SXVI and SiXIV (GRB 020813) and from 
helium/hydrogen-like Mg, Si, S, Ar and Ca (GRB 030227). Also in these cases a thermal model could fit the data, but the authors noted that these lines can also originate from reflection by material illuminated by the burst/afterglow (even though specific fits for this case are not presented for GRB 020813). Even more interestingly, in the case of GRB 030227 the lines are not detected in the first part of the observation (started about $11 \mathrm{~h}$ after the burst) but appear only in the last $10 \mathrm{ks}$, approximately $20 \mathrm{~h}$ after the trigger.

All these lines are detected at the $\sim 3 \sigma$ level: although we are waiting for a more secure detection, we assume that these lines are real, and in this paper we reanalyze the XMM-Newton data of GRB 011211 to show that it is possible to reproduce them modelling the spectrum with the reflection model, once lines from light elements are properly considered. We discuss the case in which the reflector has a funnel geometry and we propose simple observational tests for this scenario that could be performed with future missions such as SWIFT. We find a consistent scenario able to account for the observations of the emission lines of light metals (but not iron) in GRB 011211, and we point out that if this scenario is correct there should be an intense optical-UV-soft X-ray emission accompanying the emergence of the emission lines. This is therefore a strong test for the reflection interpretation of the X-ray emission lines.

In Sect. 2 we report the results of the fit with the reflection model. In Sect. 3 we derive and discuss the constraints on the GRB environment and the properties of the reflection model.

The following cosmology is assumed: $H_{0}=70 \mathrm{~km} \mathrm{~s}^{-1} \mathrm{Mpc}^{-1}$, $\Omega_{\Lambda}=0.7$ and $\Omega=1$.

\section{Spectral fits}

XMM-Newton data of GRB 011211 processed through the standard pipeline are publicly available ${ }^{1}$. We extract the PN spectrum of the first $5 \mathrm{ks}$, where lines are visible (Reeves et al. 2002, 2003), using the standard package SAS. We fitted the spectrum using XSPEC (v.11).

\subsection{Pure reflection}

Unfortunately, the reflection model available in XSPEC (available as a table model file; see the description of the model in Ballantyne et al. 2001) is calculated for two fixed values of the abundance, solar and $2 \times$ solar. Moreover, as anticipated in the Introduction, it does not include light metals such as $\mathrm{S}, \mathrm{Ar}$ and $\mathrm{Ca}$. The reflection model assumes that a slab is illuminated by a power law continuum and the basic fitting parameter is the ionization parameter, defined as $\xi=L_{\mathrm{ill}} /\left(n R^{2}\right)$, where $L_{\mathrm{ill}}$ is the luminosity of the continuum, $n$ is the density of the illuminated slab and $R$ is the distance between the slab and the illuminating source.

To include the contribution of light metals we fit the data using the following procedure: we start fitting the data by using the (absorbed with Galactic $N_{\mathrm{H}}=4.2 \times 10^{20} \mathrm{~cm}^{-2}$, Reeves et al. 2003) reflection model with free redshift. In the fitting procedure we excluded all the bins below $0.3 \mathrm{keV}$, due to

\footnotetext{
${ }^{1}$ http://xmm.vilspa.esa.es/external/xmm_sched/too
}

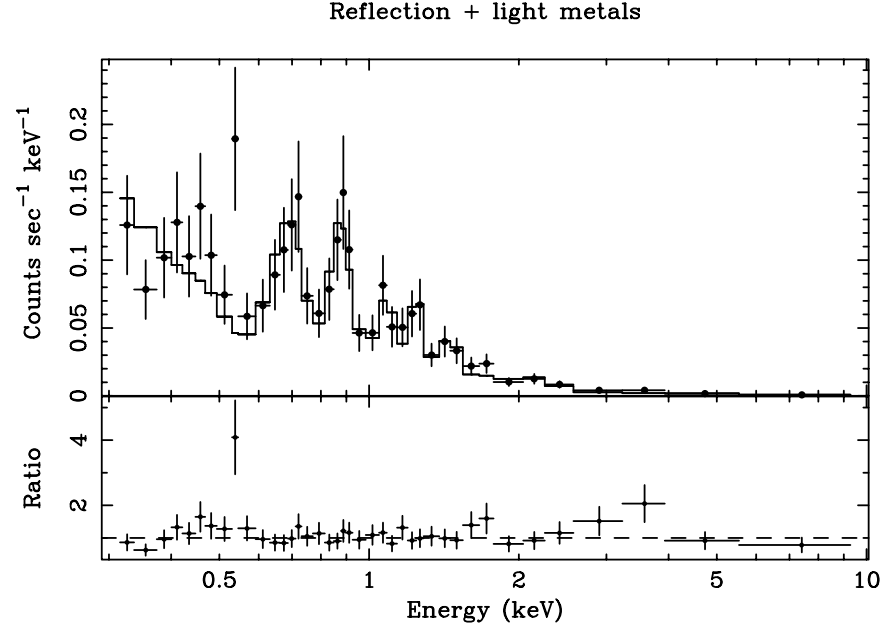

Fig. 1. Spectrum (top panel) and data/model ratio (bottom panel) of the first $5 \mathrm{ks}$ of the PN data fitted with the reflection model and lines from light elements (see discussion in the text).

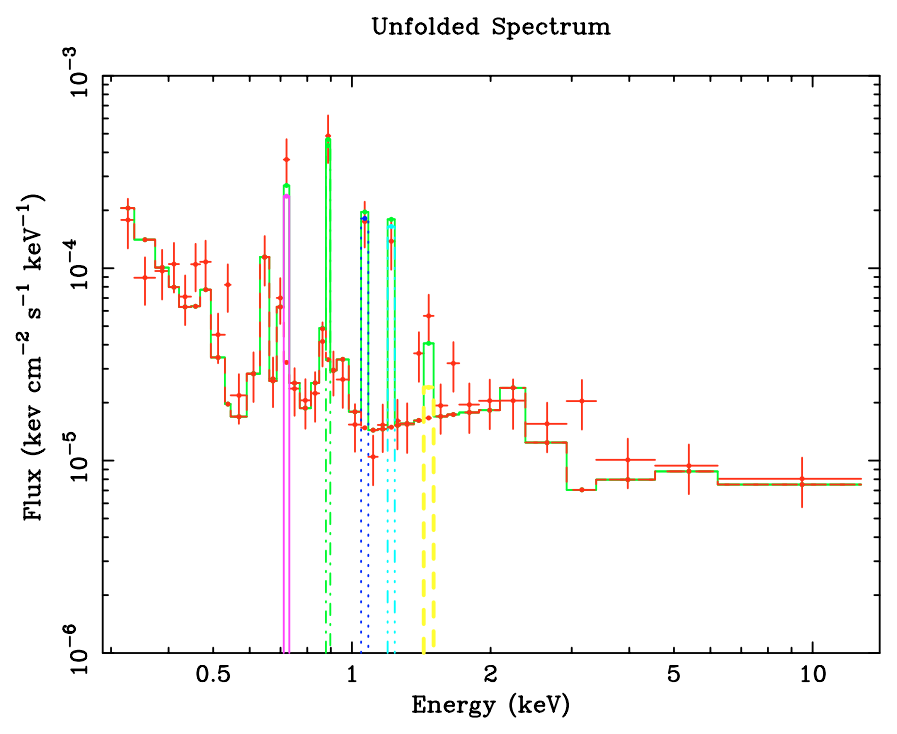

Fig. 2. Unfolded spectrum of the PN spectrum fitted with the reflection model. The narrow lines at $0.7,0.9,1.1,1.2$, and $1.5 \mathrm{keV}$ are the lines of the elements reported in Table 1, not included in the reflection model.

known problems with the calibration of the response matrix (e.g. Brinkmann et al. 2001). As expected, the residuals of the best fit (which converges at $z=1.9$ ) show clear deviations around $0.7,0.9,1.1,1.2$ and $1.5 \mathrm{keV}$. This agrees with the analysis by Reeves et al. The line at $0.7 \mathrm{keV}$ can be attributed to SiXIV. The model includes this element, but the fit of the line is poor, since the data requires a brighter line (which may correspond to a larger abundance). The other features can be identified as emission lines from elements not included in the reflection model (SXVI at $0.9 \mathrm{keV}$, ArXVII and SXV at $1.1 \mathrm{keV}$, Ar XVIII at $1.2 \mathrm{keV}$ and CaXX at $1.5 \mathrm{keV}$ ). We then added Gaussian emission lines to account for these residuals. The folded spectrum and the model/data ratio are shown in Fig. 1. The unfolded spectrum is shown in Fig. 2. The best fit $\left(\chi^{2} /\right.$ d.o.f. $\left.=27.45 / 26\right)$ parameters are: $\log \xi=2.25 \pm 0.30$, 
Table 1. Parameters of the lines added to the reflection model (see text for details). Luminosities refer to the pure reflection case; in the reflection+power-law case they have to scaled by a factor 0.7 .

\begin{tabular}{lllll}
\hline \hline Ion & $\begin{array}{l}E_{\text {obs }} \\
\mathrm{keV}\end{array}$ & $\begin{array}{l}E_{\text {rest }} \\
\mathrm{keV}\end{array}$ & $z$ & $\begin{array}{l}L \\
10^{45} \mathrm{erg} \mathrm{s}^{-1}\end{array}$ \\
\hline SiXIV & 0.71 & 1.99 & 1.82 & 0.71 \\
SXVI & 0.88 & 2.60 & 1.94 & 1.2 \\
ArXVII (SXV) & 1.08 & $3.16(3.14)$ & $1.93(1.91)$ & 0.95 \\
ArXVIII & 1.23 & 3.30 & 1.73 & 0.95 \\
CaXX & 1.50 & 4.07 & 1.71 & 0.23 \\
\hline
\end{tabular}

$z=1.93 \pm 0.10$ with an incident power-law of photon spectral index $\Gamma=2.43 \pm 0.20$.

The parameters of the lines we have added, but that are not included in the fitting model, are reported in Table 1. Similarly to Reeves et al. (2003) we find that the lines require a redshift $z=1.8-1.9$, lower than the redshift of the GRB, $z=2.140 \pm 0.001$ (Holland et al. 2002), suggesting that the emitting material is moving with a velocity $v \sim 0.1 c$. The luminosity of the Fe line included in the model is $2.3 \times 10^{44} \mathrm{erg} \mathrm{s}^{-1}$. For the possible presence of the H-like nickel (rest frame energy $E=8.102 \mathrm{keV}$ ) we obtained an upper limit to the equivalent width of $E W<450 \mathrm{eV}$ (Reeves et al. 2003 found $E W<$ $800 \mathrm{eV}$ ). The expected luminosities of light elements, compared to iron, have been calculated by Lazzati et al. (2002b). The maximum contrast between the luminosity of the lines of the light metals and the iron line is reached around $\xi \sim 10^{2}$. For $\xi \sim 2 \times 10^{2}$, required by our fit, we expect a luminosity ratio of the order of 3 , in agreement with the luminosities reported in Table 1. Looking in detail at each element we note that lines of $\mathrm{S}$ and Ar appear to be more luminous than expected, by a factor of 2 and 5, respectively (note however that, due to the relatively large uncertainties, the normalizations are consistent with solar abundance within $1 \sigma$ and $2 \sigma$, respectively). This discrepancy (present also in the fits presented by Reeves at al.) could be ascribed to a larger abundance of these elements with respect to the solar value.

Thus we can conclude that, once light metals are included, the reflection model provides a consistent fit of the $X M M-N e w t o n$ spectrum.

\subsection{Reflection plus underlying power-law}

Although a pure reflection model can account for the observed $\mathrm{X}$-ray spectrum, it is possible, and even likely, that part of the flux originates from the afterglow continuum. It is important to stress that, unlike the case of AGNs, the continuum reprocessed through the reflection and that directly observed from the afterglow, simultaneously with the lines, could be different. In fact the radiation reprocessed by the reflector originates from the unobserved, rapidly changing burst/early-afterglow phase (hereafter illuminating continuum), while the afterglow radiation (observed when lines are detected) is produced much later (hereafter late afterglow continuum).

We performed a series of fits to asses how much of the total emission can be ascribed to an underlying power-law continuum produced by the late afterglow continuum. We fit the data with a model including a power-law and the reflection spectrum. We fix the relative normalization of the reflection spectrum and of the Gaussian lines to the value found in the fit with the pure reflection case.

First we constrain the two slopes, that of the illuminating continuum and that of the late afterglow power-law, to the same value. The fit converges to $\Gamma=2.26 \pm 0.1, \log \xi=1.9 \pm 0.1$ with $\chi^{2} /$ d.o.f. $=23.71 / 31$. The significantly improves with respect to the pure reflection case. The presence of a direct power-law is therefore suggested by the data.

We then allowed the two photon indices to vary independently. In this case the best fit parameters are: $\Gamma_{\mathrm{pl}}=2.04_{-0.08}^{+0.29}$, $\Gamma_{\text {refl }}=2.39_{-0.06}^{+0.1}, \log \xi=2.1 \pm 0.1$ with $\chi^{2} /$ d.o.f. $=23.13 / 30$. Within the $90 \%$ conf. level, the two slopes are consistent. Reflection contributes for $\sim 70 \%$ of the flux. Correspondingly, the luminosity of the emission lines given in Table 1 decreases by a factor 0.7 .

\section{Discussion}

We showed that the XMM-Newton PN data of GRB 011211 can be satisfactorily fitted by the pure reflection model, if lines from the elements not included in the spectral model are properly considered. The ionization parameter $\xi$ is well constrained to be of the order of $10^{2}$, since the data do not present a prominent iron line while lines of light metals are luminous. As mentioned, the absence of a prominent iron line is naturally predicted when the ionization parameter $\xi$ is around $10^{2}$ (Lazzati et al. 2002b). In this case, due to the importance of the Auger effect, the emission from iron is strongly depressed compared to the other metals.

The reflection model can explain very different spectra, since variations of $\xi$ in different bursts could produce different reflection spectra, with prominent or almost absent lines (e.g. Ballantyne \& Ramirez-Ruiz 2001). Low values of the ionization parameter $(\xi<100)$ will produce a reflection component with evident lines. A large ionization parameter $\left(\xi>10^{4}\right)$, on the other hand, would produce almost featureless spectra, with a shape similar to the shape of the illuminating continuum. For a narrow range of $\xi$ centered around $10^{2}$ the spectra will show luminous lines from light metals and a depressed $\mathrm{K}_{\alpha}$ iron line. It may be argued that in the case of GRBs a well defined ionization parameter $\xi$ can hardly be attained. In the geometry we consider here, the observer receives a spectrum resulting from the convolution of reflected components with different illuminating spectra. Regions closer to the line of sight will contribute low $\xi$ components, while regions away from the line of sight will contribute higher $\xi$ components, since the time-delay is longer and the ionizing continuum is a monotonically decreasing function (at least in the afterglow phase). While this is true, it is clear that one of the components (and hence a well defined $\xi$ ) will dominate. Let us now consider the early X-ray afterglow as the illuminator, since the GRB proper will have a too high $\xi$ to produce lines, as discussed above. If the illuminator has a power-law decay in time $L \propto t^{-\alpha}$, the more (less) luminous phase will dominate the observed reflected component for $\alpha>1(\alpha<1)$. Unfortunately the X-ray afterglow has never been observed early enough to constrain its decay 


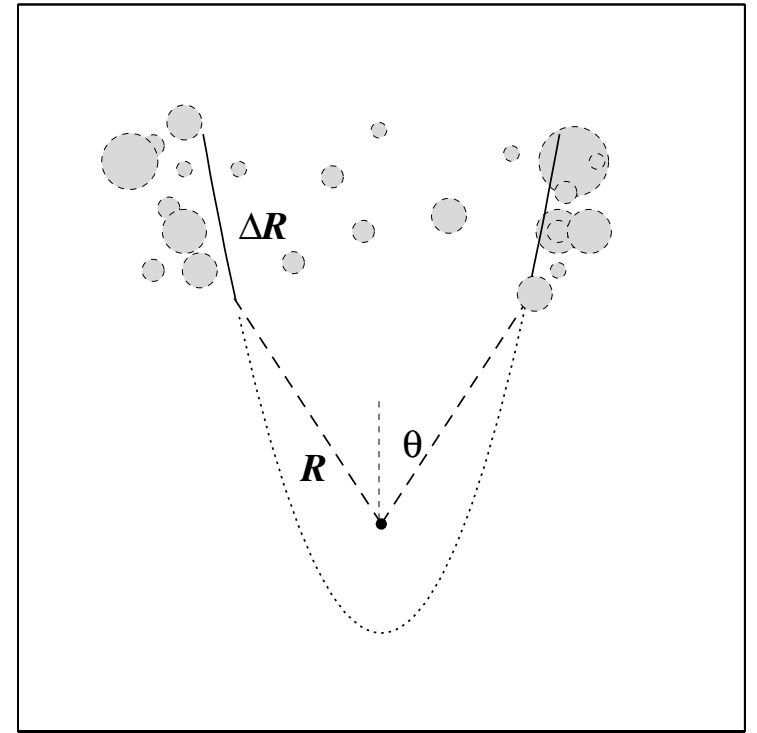

Fig. 3. Sketch of the geometry assumed for the reflecting material (not to scale). The material is organized in clumps along the walls of the funnel (assumed to have a parabolic shape). Clumps could also lie along the line of sight.

in the early phases, but it is not unreasonable to assume that the X-ray band lies initially below the cooling frequency, and that its decay slope is initially the same as the optical slope at later stages. In this case, bursts with an initially shallow decay in the optical, such as GRB 011211 (Holland et al. 2002) and GRB 020813 (Covino et al. 2003), should show low-ionization features, while bursts with initial fast decay such GRB 991216 (Halpern et al. 2000) should have X-ray spectra with strong iron lines. Even though it is not possible to make statistics with such a handful of cases, it is tantalizing that such a correlation is indeed respected in all cases thus far.

In the following we derive the geometrical and physical setup of the reflecting material. We note that the actual geometry of the funnel is unimportant for most of the estimates reported below, aiming to find some basic quantities such as the overall size and the total mass of the line emitting material. The emitting layer is supposed to be illuminated by an intense $\mathrm{X}$-ray flux: this requires that either the illuminating source is less collimated than the funnel (if it has a conical profile), or that the funnel deviates from a perfect conical geometry, being e.g. parabolic. In these conditions the funnel walls can receive a non-negligible fraction of the ionizing flux. We then consider more complex geometries, involving clumps of overdense material, possibly located close to the funnel surface, as illustrated in Fig. 3.

\subsection{General constraints}

Once the ionization parameter and the line luminosities are known, we can infer important clues to the physical state of the reflector. The "observed" or fixed quantities are the following:

1. the luminosity of the line $L_{\text {line }}$;

2. its duration $t_{\text {line }}$;
3. the time $t_{\text {app }}$ at which the line starts to be visible with the luminosity $L_{\text {line }}$

4. the ionization parameter $\xi$;

5. the efficiency $\eta$ of converting the illuminating continuum into the specific line;

6. the total mass $M_{\mathrm{T}}$ (which we require not to exceed $\left.\sim 10 M_{\odot}\right)$.

Note that the time at which lines appear $\left(t_{\text {app }}\right)$ is an observable in principle, but it may require early observations of the X-ray afterglow to be detected. Indeed, it has been already determined only in one case, (GRB 030227) while we have only an upper limit for all other cases. However, it is likely that with a short pointing time, like the one provided by SWIFT, the number of cases in which this parameter will be observable will strongly increase. Therefore, for a general discussion, we suppose that $t_{\text {app }}$ is known. For the specific case of GRB 011211 we only have an upper limit to $t_{\text {app }}$, since the lines were detected in the first part of the observation.

Unknown physical quantities of the system are:

1. the distance $R$ between the illuminator and the line emitting material;

2. the collimation angle $\theta$ of the illuminator (see Fig. 3);

3 . the electron density $n_{\mathrm{e}}$ (which, for the large ionization conditions discussed here, is close to the proton density $n_{\mathrm{p}}$ );

4. the illuminating energy $E_{\mathrm{ill}}$ (or, alternatively, the illuminating luminosity $L_{\text {ill }}$ ) directly intercepted by the line-emitting material. Part of this illuminating energy can come from the burst emission, and part from the early afterglow. It is a rapidly variable quantity, and for the sake of simplicity we will assume that $L_{\text {ill }}$ is an appropriate average over $t_{\text {ill }}$;

5. the time $t_{\text {ill }}$ for which the illumination lasts;

6 . the number of line photons, $k$, produced by each atom during $t_{\text {ill }}$;

7. the filling factor $f$;

8. the height of the shell $\Delta R$.

Thus we have a total of 6 quantities provided by observations and 8 unknown quantities. Therefore we will consider $\theta$ and $t_{\text {ill }}$ as free parameters, even if their values are limited (for instance, "reasonable" aperture angles of the illuminator should be between 10 and 60 degrees; the illuminating time should be shorter than the time for which the line is visible, $\left.t_{\text {ill }}<t_{\text {line }}\right)$.

In the following we outline how we can connect these two sets of parameters and how we can derive the parameters of the system by the observational quantities. All the time intervals $\left(t_{\text {ill }}, t_{\text {line }}, t_{\text {app }}\right)$ are intended as measured in the frame of the burst. We adopt the notation $Q \equiv 10^{x} Q_{x}$ and use cgs units.

The size of the reflector is related to the time at which lines become visible:

$R=\frac{c t_{\text {app }}}{1-\cos \theta}$

The total mass of the element emitting a specific line (denoted by the subscript $i$ ) is related to the number $N_{i}$ of photons in that line by:

$M_{i}=\frac{N_{i} W_{i} m_{\mathrm{p}}}{k}$ 
where $W_{i}$ is the atomic weight of the element. $k$ is the number of line photons a single atom can emit during the illumination phase (Lazzati et al. 1999):

$k=\frac{t_{\text {ill }}}{t_{\text {rec }}}$

where the recombination time is:

$t_{\text {rec }}=\frac{1}{\alpha_{\mathrm{r}} n_{\mathrm{e}}} \mathrm{s}$.

$n_{\mathrm{e}}$ is the numerical density of the free electrons and $\alpha_{\mathrm{r}}$ is:

$\alpha_{\mathrm{r}}=5.2 \times 10^{-14} Z \lambda^{1 / 2}\left[0.429+0.5 \ln (\lambda)+\frac{0.496}{\lambda^{1 / 3}}\right]$

where $\lambda=1.58 \times 10^{5} Z^{2} T^{-1}, Z$ the atomic number of the element and $T$ the electron temperature.

The total number of photons produced by atoms of the element $i$ can be written as:

$N_{i}=\frac{E_{\text {line }}}{\epsilon_{i}}=\frac{L_{\text {line }} t_{\text {line }}}{\epsilon_{i}}$

where $E_{\text {line }}=L_{\text {line }} t_{\text {line }}$ is the total energy in the line and $\epsilon_{i}$ is the energy of the line. The mass of the layer emitting the observed line is (using the mass abundance $A_{i}$ of the emitting element):

$M_{1}=\frac{M_{i}}{A_{i}}=\frac{L_{\text {line }} t_{\text {line }} W_{i} m_{\mathrm{p}}}{k A_{i} \epsilon_{i}}$.

This mass is contained into a layer of volume $V$, of thickness $\Delta x$, radius $R \sin \theta$ and height $\Delta R$. The material reflecting the illuminating continuum and producing the line is contained in a layer of height $\Delta x$ of unit scattering optical depth: $\tau_{\mathrm{T}}=\sigma_{\mathrm{T}} n_{\mathrm{e}} \Delta x=1$. Since the matter is highly ionized the density $n_{\mathrm{e}}$ of free electrons is the same as the proton density $n_{\mathrm{p}}$. This allows us to derive the mass of this layer in a way independent of Eq. (7) and also independent of the electron density:

$$
\begin{aligned}
M_{1} & =n_{\mathrm{p}} m_{\mathrm{p}} V=2 \pi R \sin \theta \Delta R \Delta x n_{\mathrm{e}} m_{\mathrm{p}} \\
& =\frac{2 \pi}{\sigma_{\mathrm{T}}} R \Delta R \sin \theta m_{\mathrm{p}} \\
& \simeq 5 \pi R \Delta R \sin \theta \mathrm{g} .
\end{aligned}
$$

We then have two independent estimates of the mass involved in the line production, Eqs. (7) and (8). By equating them we can derive the value of $k$ (which becomes independent of $n_{\mathrm{e}}$ )

$k=\frac{W_{i} m_{\mathrm{p}}}{A_{i} \epsilon_{i}} \frac{L_{\text {line }} t_{\text {line }}}{5 \pi R \Delta R \sin \theta}$.

From the definition (3) and Eq. (4) and inserting $k$ from (9) we can derive the density:

$$
\begin{aligned}
n_{\mathrm{e}} & =\frac{k}{\alpha_{\mathrm{r}} t_{\mathrm{ill}}} \\
& =\frac{W_{i} m_{\mathrm{p}}}{A_{i} \epsilon_{i}} \frac{L_{\text {line }} t_{\text {line }}}{5 \pi R \Delta R \sin \theta \alpha_{\mathrm{r}} t_{\mathrm{ill}}} \mathrm{cm}^{-3} .
\end{aligned}
$$

The ionization parameter is defined as:

$\xi=\frac{L_{\mathrm{ill}}}{n_{\mathrm{e}} R^{2}}$
Inserting the density given by Eq. (10), we have:

$$
L_{\text {ill }}=\frac{1}{5 \pi} \xi \frac{R}{\Delta R} \frac{W_{i} m_{\mathrm{p}} E_{\text {line }}}{A_{i} \epsilon_{i} \sin \theta \alpha_{\mathrm{r}} t_{\mathrm{ill}}} \operatorname{erg~s}^{-1}
$$

The efficiency $\eta$ in producing the line is defined as:

$\eta=\frac{E_{\text {line }}}{E_{\text {ill }}}=\frac{L_{\text {line }} t_{\text {line }}}{L_{\text {ill }} t_{\text {ill }}}$.

Using Eqs. (12) and (13) we derive:

$\frac{\Delta R}{R}=\frac{1}{5 \pi} \xi \eta \frac{W_{i} m_{\mathrm{p}}}{A_{i} \epsilon_{i} \sin \theta \alpha_{\mathrm{r}}}$.

From Eq. (8) the mass of the layer turns out to be:

$M_{1}=R^{2} \xi \eta \frac{W_{i} m_{\mathrm{p}}}{A_{i} \epsilon_{i} \alpha_{\mathrm{r}}} \quad \mathrm{g}$.

The total amount of mass $M_{*}$ (i.e. contained in a shell of surface $4 \pi R^{2}$ and height $\Delta R$ and assuming the same density) will be:

$M_{*}=n_{\mathrm{p}} m_{\mathrm{p}} V_{*}=4 \pi R^{3} \frac{\Delta R}{R} n_{\mathrm{e}} m_{\mathrm{p}}$.

We impose that this mass cannot exceed $M_{\mathrm{T}} \sim 10 M_{\odot}$. If $M_{*}$ exceeds this value, we assume that the material is distributed inhomogeneously, more dense in the walls of the funnel or organized in single clumps (see below). We parametrize the deviation from the homogenoeus case by the filling factor:

$f=\frac{M_{\mathrm{T}}}{M_{*}}$

Note that there is in principle another equation relating $\Delta R$ with the duration of the line $t_{\text {line }}$ given by

$\Delta R \sim \frac{c t_{\text {line }}}{\cos \theta}$

This equation is valid only in the case in which the duration of the emission of the line is dominated by geometrical effects, i.e. $t_{\text {ill }}<\Delta R / c$. In principle, in this case it is possible to fix the aperture angle of the funnel, using Eq. (14) together with Eqs. (18) and (1):

$\tan \theta(1-\cos \theta) \sim \frac{1}{5 \pi} \xi \eta \frac{W_{i} m_{\mathrm{p}}}{A_{i} \epsilon_{i} \alpha_{\mathrm{r}}} \frac{t_{\text {app }}}{t_{\text {line }}}$.

For the application to the specific case of GRB 011211 we have preferred to use the angle $\theta$ as a free parameter, using Eq. (18) as a consistency check. We do that because of the uncertainties related to the specific geometry.

We stress that the above estimates have been derived for a specific geometry, i.e. a uniform funnel over-dense with respect to the rest of the remnant. It is conceivable that the real situation is much more complex: for instance, dense clumps of matter could be embedded in a less dense medium, and be located also in the interior of the funnel, not only on its walls (Fig. 3). On the other hand the values of the parameters we derive will not dramatically change in this case, and can therefore be thought as indicative numbers. 


\subsection{Application to GRB 011211}

As an illustrative example of the application of the estimates given above we can apply our treatment to the specific case of GRB 011211. For the observational quantities we set:

- $L_{\text {line }}=7 \times 10^{44} \mathrm{erg} \mathrm{s}^{-1}$ for the SiXIV line;

$-t_{\text {line }}=5 \times 10^{3} /(1+z) \sim 1.7 \times 10^{3} \mathrm{~s}$;

$-t_{\text {app }} \leq 4 \times 10^{4} /(1+z) \sim 1.3 \times 10^{4} \mathrm{~s}$;

$-\xi=10^{2} \xi_{2}$

- $\eta=10^{-2} \eta_{-2}$ (see Ghisellini et al. 2002);

- $M_{\mathrm{T}}=10 M_{\odot}$.

In addition, we have used the redshift $z=2.14$; the line energy $\epsilon_{\text {SiXIV }}=1.99 \mathrm{keV}$ and an abundance $A_{i}$ equal to twice the solar value. The temperature of a photoionized plasma illuminated with $\xi \sim 100$ is predicted to be in the range $10^{5}-10^{6} \mathrm{~K}$ (e.g. Kallman \& McCray 1982). We have then assumed that the line emitting material is at $T=5 \times 10^{5} \mathrm{~K}$. A variation of the temperature, directly affecting the recombination rate, implies a different amount of matter necessary to account for the emission and therefore the estimate of the density and of the clumping factor. Equation (5) indicates that the dependence of $t_{\text {rec }}$ on the temperature is $t_{\mathrm{rec}} \sim T^{3 / 4}$. A larger (lower) temperature would decrease (increase) the recombination rate, resulting in a larger (lower) clumping $f \sim T^{-3 / 4}$.

As discussed in Sect. 3.1, in the specific case of GRB 011211, since the appearence time of the line is unknown, the value of $t_{\text {app }}$ given above should be considered an upper limit to the actual value and, therefore, the estimates given below should be considered as limits. In particular the derived distance $R$ is un upper limit, the density is a lower limit and thus the clumping factor should be considered as a lower limit. In Fig. 4 we show a few derived quantities as a function of the aperture angle $\theta$ and for $t_{\text {ill }}=300$ and $1000 \mathrm{~s}$. The distance $R$ is of the order of a few times $10^{15} \mathrm{~cm}$; the density $n_{\mathrm{e}}$ is of the order of $10^{14}-10^{15} \mathrm{~cm}^{-3} ; \Delta R / R$ is of the order of a tenth, and the filling factor of the order of $f \sim 10^{-3}$. The mass of the line emitting layer is $M_{1}=10^{-4}-10^{-2} M_{\odot}$. It is interesting to note that the assumed (admittedly simple) geometry can also account for the line duration, which yields an independent value of $\Delta R / R$ (dashed line in the top panel of Fig. 4). According to it, the illumination angle turns out to be $\sim 45^{\circ}$ (i.e. where the dashed and solid lines labeled as $\Delta R / R$ cross).

The portion $E_{\mathrm{ill}}$ of the total energy of the illuminating continuum directly impinging on the reflecting matter will be

$E_{\text {ill }} \simeq \frac{1}{\eta} L_{\text {line }} t_{\text {line }} \simeq 3.5 \times 10^{50} \mathrm{erg}$.

The total apparent isotropic energy contained in the illuminating component will be $E_{\text {iso }}=E_{\text {ill }} / \Delta \Omega$, where $\Delta \Omega$ is the solid angle subtended by the reflecting matter as observed at the location of the illuminating source. It can be shown that in the geometry assumed here and with the geometrical parameters found above $\Delta \Omega \sim 0.014 \pi$, giving $E_{\text {iso }} \simeq 3 \times 10^{51} \mathrm{erg}$, consistent with the early phase of the afterglow.

We conclude that the reflection model offers a good interpretation of the data. The reflecting material must be clumped,

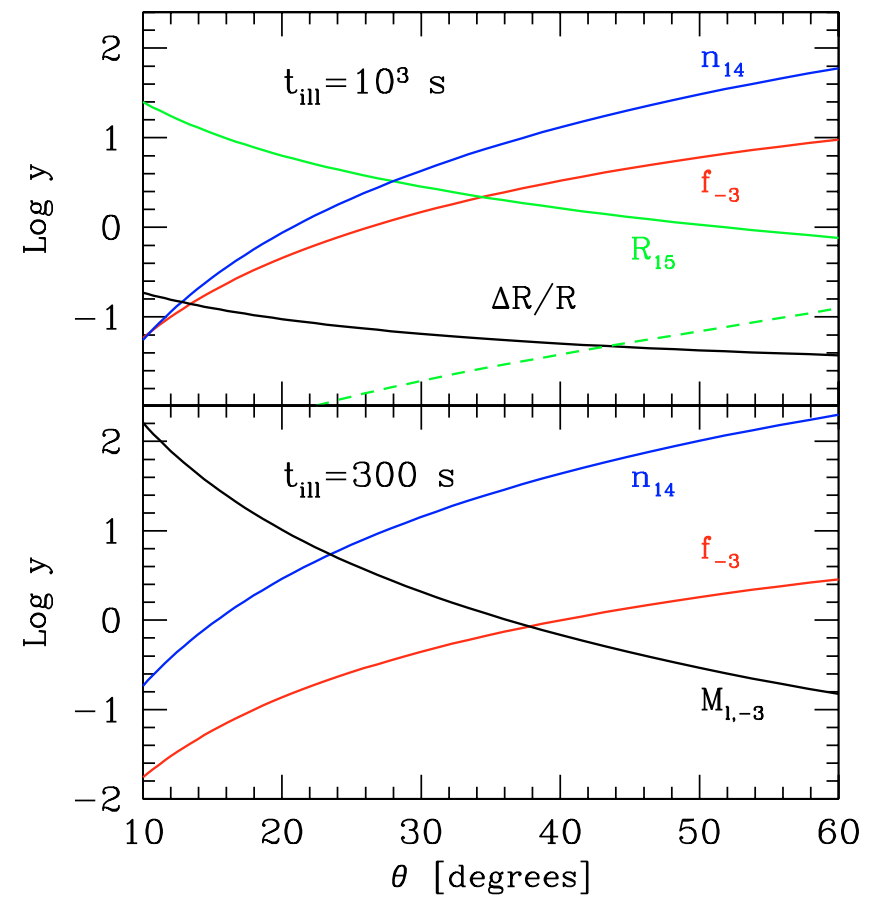

Fig. 4. Derived quantities for GRB 021211, for $t_{\text {ill }}=10^{3} \mathrm{~s}$ (top panel) and for $t_{\text {ill }}=300 \mathrm{~s}$ (bottom panel). The filling factor is in units of $10^{-3}$, the distance $R$ in units of $10^{15} \mathrm{~cm}$, the density $n$ in units of $10^{14} \mathrm{~cm}^{-3}$ and the mass of the emitting layer in units of $10^{-3}$ solar masses. The quantities $R, \Delta R / R$ and $M_{1}$ do not depend on $t_{\text {ill }}$. The dashed line in the top panel is the value of $\Delta R / R$ derived using Eqs. (1) and (18). See text for the assumed values of the input parameters.

although the required clumping factor is smaller (and the corresponding filling factor greater) than the one required by the thermal model (Lazzati 2003).

\subsection{Re-emission of absorbed radiation}

An important consequence of the assumption that reflection is responsible for the production of detected lines is that, especially when $\xi$ is low $\left(\xi<10^{2}\right)$ as in the case discussed here, most of the incoming radiation is absorbed by the reflecting material and successively re-emitted as low-frequency radiation.

In the low-ionization condition discussed here the reflected luminosity is $\sim 10 \%$ of the total incident luminosity (e.g. Zicky et al. 1994): therefore the remaining $\sim 90 \%$ is absorbed by the medium, which is heated-up and re-emits what it has absorbed. The luminosity $L_{\text {repr }}$ of this reprocessed component is rather high and amounts to:

$L_{\text {repr }}=0.9 \frac{L_{\text {line }}}{\eta} \sim 9 \times 10^{46} \frac{L_{\text {line }, 45}}{\eta_{-2}} \quad \mathrm{erg} \mathrm{s}^{-1}$.

This emission lasts exactly for the same time as the X-ray emission line, and is therefore a powerful diagnostic of the reflection model. The above luminosity, for the case of GRB 011211 at $z=2.14$, corresponds to a flux of $3 \times 10^{-12} \mathrm{erg} \mathrm{s}^{-1} \mathrm{~cm}^{-2}$. The problem is to assess the spectral region where this emission peaks. We should solve the radiation transfer into a slab, heated by the incoming rapidly variable high energy emission from the 
early afterglow. The surface of the slab, directly illuminated by the incoming continuum, will be heated to high temperatures, close to the Compton temperature $T_{\mathrm{C}} \sim 10^{7} \mathrm{~K}$. The emission from these outer portion will peak in the X-ray region, at energies around $1 \mathrm{keV}$. Gas in the layers deeper in the slab will be close to the thermal equilibrium: therefore the temperature of these region will be close to the black-body temperature corresponding to a black-body emitting a luminosity of the order of $L_{\mathrm{bb}}=L_{\mathrm{repr}}$ :

$T_{\mathrm{bb}} \simeq\left(\frac{L_{\mathrm{bb}}}{\sigma A}\right)^{1 / 4} \simeq 2 \times 10^{5}\left(\frac{L_{\mathrm{repr}, 47}}{A_{30}}\right)^{1 / 4} \mathrm{~K}$

where the area $A=2 \pi R \sin \theta \Delta R$ has been assumed to be of the order of $A=10^{30} A_{30} \mathrm{~cm}^{2}$. The maximum of the emission falls at the frequency $v_{\mathrm{bb}}=3 k T /[(1+z) h] \sim 10^{15} \mathrm{~Hz}$, i.e. in the near UV.

The actual outcoming spectrum will be a complex integral over the emission from the different layers (weighted by the optical depth of the layer), in strongly time-dependent conditions. The detailed calculation of the spectrum is beyond the scope of this paper. Moreover, note that the conditions appropriate to analyze even a steady state case are at the limit of the calculation possibility of the program CLOUDY (Ferland 1996) which gives results that are not completely reliable.

What we can safely conclude is that, simultaneously with the presence of the X-ray emission lines from light metals, there should be a bright component between the UV and the soft X-rays, which has a luminosity $\sim 100$ times larger than the line luminosities (and 10 times larger than the entire X-ray reflection continuum). The low frequency tail of this reprocessed component falls in the optical band, where it can be observed as a rebrightening of the light curve. As an example, if 1 per cent of the reprocessed luminosity is emitted in the optical $V$-band, we should see a magnitude of $V \sim 22$.

We stress that the presence of the reprocessed emission is a natural consequence of the reflection interpretation: observations in the UV-soft X-ray bands, confirming or ruling out the existence of this component accompanying the X-ray emission lines provide a powerful and reliable diagnostic tool to study the origin of the emission features. The only way we envisage not to see this reprocessed component in the reflection scenario (with a small ionization parameter) is to have dense and small clumps with a Thomson optical depth close to unity. These blobs would efficiently reflect the incoming continuum, but only a small fraction of it is absorbed and re-emitted. Since this requires a rather ad-hoc setup, we consider it unlikely.

\subsection{Close reprocessor}

We discussed the case of a far reprocessor, located at a distance $R \sim 10^{15}-10^{16} \mathrm{~cm}$ from the illuminator. There is however the possibility that the material producing the lines is located very close to the central source. In this case the duration of the line is linked to the time for wich the central engine is active. A model of this type has been proposed by Rees \& Mészáros (2000) and Mészáros \& Rees (2001).

The discussion given in the previous sections can be easily applied to this different scenario. In this case Eq. (1) can be dropped, since the geometrical effects are not important in determining the time at which the lines appear. All the other equations still apply, once $\theta$ is fixed to $\pi / 2$. In this way we can model a cilindrical geometry, with the illuminating source located on the axis. We fixed the size to $R=10^{13} \mathrm{~cm}$, the typical size considered by Rees \& Mészáros (2000) and Mészáros \& Rees (2001).

We can derive the interesting physical parameters for the value of the observational quantities given above in the same way. We found: $f=4, n_{\mathrm{e}}=1.2 \times 10^{20} \mathrm{~cm}^{-3}, \Delta R / R=0.03$ and $M_{1}=2.5 \times 10^{-8} M_{\odot}$. The large value of the density is a consequence of the fact that, for a fixed value of the ionization parameter, Eq. (11) implies a large density for compact (i.e. small $R$ ) reprocessor. The value of $f$ (close to 1 ) shows that no clumping is needed in this case, due to the smaller quantity of iron required compared to the far reprocessor case.

Although the values of the derived physical quantities are reasonable, this version of the reflection scenario suffers from many problems. First (e.g. Lazzati et al. 2002b), the lines and the continuum responsible for the lines are observed together: thus it is not possible to produce lines with the large observed $E W$ (hundreds of $\mathrm{eV}$ ). This problem is naturally by-passed by the far-reprocessor scenario, since, due to the crossing time delay, the lines are observed much later than the direct continuum. A possibility to solve the difficulty in the close reprocessor is to admit that the illuminating continuum is strongly anisotropic and threfore it is not directly observable.

Another problem is related to the high temperature reached by the illuminated material. Following the results of Sect. 3.3, the temperature of the material will lie between $T_{\mathrm{bb}}$ and $T_{\mathrm{C}}$. Due to the small area, $A \simeq 10^{25} \mathrm{~cm}^{2}$, Eq. (22) gives $T_{\mathrm{bb}} \sim$ $3.5 \times 10^{6} \mathrm{~K}$. The reprocessed thermal radiation will therefore peak in the soft X-ray domain $(>0.1 \mathrm{keV})$. A strong soft excess has never been observed in the X-ray spectra of afterglows with lines.

For these reasons we consider this possibility unlikely.

\subsection{Time evolution}

All the issues related to the GRB are characterized by a strong time-dependent phenomenology. Besides the normal afterglow behavior produced by the decelerating fireball, in the X-ray band we have the possibility to see the reflection component, which will be variable in flux and possibly in shape. The visibility of the reflected component with the associated emission lines depends upon the relative strength of the afterglow and of the reflected component, and on the ionization parameter determining which lines are most efficiently produced. In general, the reflection component will be visible (if at all) only in intermediate phases of the afterglow: on the one hand the luminosity of the afterglow must decrease sufficiently to allow the reflection to be observed, but on the other hand the reflected radiation can be observed only for a time smaller than the light travel time of the line emitting material (after that time we expect the reflection component to disappear).

At these intermediate times, if the reflection component contributes to the total X-ray flux, it will produce a "bump" 
in the X-ray lightcurve, flagging the likely presence of lines in the X-ray spectrum. At the same time, we expect a change in the slope of the X-ray spectrum, which will depend upon the ionization parameter. In fact, for relatively small $\xi$, the reflecting layer will not be completely ionized, and the reflection component will be hard (i.e. characterized by a photon spectral index $\Gamma<2$ ) in the $0.1-10 \mathrm{keV}$ band, while for large $\xi$ the reflecting layer will be almost completely ionized, and the shape of the reflected component would closely resemble the shape of the illuminating continuum. These simple arguments offer a direct test of the reflection scenario: suppose that the $\mathrm{X}$-ray light curve, after a few hours, flattens: if this flattening is due to the emergence of the reflection component then its spectrum should be harder or almost equal to the spectrum of the "normal" afterglow. If it is harder we expect a small ionization parameter and the presence of emission lines from light metals (and weak or absent lines from iron, cobalt or nickel). If the spectrum maintains a constant or quasi-constant shape, then we expect the presence of $\mathrm{K}_{\alpha}$ iron (or cobalt o nickel) lines and no emission from the fully ionized light metals, unless the ionization parameter is so large as to maintain also the iron fully ionized.

In conclusion we have presented a coherent scenario in which the emission of lines from light metals can be explained as due to the reflection by dense matter around the GRB. This scenario can be tested by observations. In particular the prediction of a bright optical-UV component coming together with the appearence of the emission lines offers a strong test for the overall picture.

Acknowledgements. We thank the referee for helpful criticisms that help us to improve the paper. We thank the Italian MIUR and ASI for financial support.

\section{References}

Antonelli, L. A., Piro, L., Vietri, M., et al. 2000, ApJ, 545, L39

Ballantyne, D. R., Iwasawa, K., \& Fabian, A. C. 2001, MNRAS, 323, 506
Ballantyne, D. R., \& Ramirez-Ruiz, E. 2001, ApJ, 559, L83

Borozdin, K. N., \& Trudolyubov, S. P. 2003, ApJ, 583, L57

Böttcher 2002, Solicited review at the 34th COSPAR Scientific Assembly, Adv. Sp. Res., submitted [astro-ph/0212034]

Brinkmann, W., Sembay, S., Griffiths, R. G., et al. 2001, A\&A, 365, L162

Butler, N. R., et al. 2003, ApJ, submitted [astro-ph/0303539]

Covino, S., Malesani, D., Tavecchio, F., et al. 2003, A\&A, 404, L5

Ferland, G. J 1996, Hazy, a brief introduction to Cloudy 94.00, Univ. Kentuky Dept. Phys. Astron. Int. Rep.

Ghisellini, G., Lazzati, D., Rossi, E., \& Rees, M. J. 2002, A\&A, 389, L33

Halpern, J. P., Uglesich, R., Mirabal, N., et al. 2000, ApJ, 543, 697

Holland, S. T., Soszyński, I., Gladders, M. D., et al. 2002, AJ, 124, 639

Kallman, T. R., \& McCray, R. 1982, ApJS, 50, 263

Lazzati, D., Campana, S., \& Ghisellini, G. 1999, MNRAS, 304, L31

Lazzati, D. 2002a, Invited talk at the meeting: Gamma Ray Bursts in the afterglow era - Third workshop. September 2002, Rome [astro-ph/0301317]

Lazzati, D., Ramirez-Ruiz, E., \& Rees, M. J. 2002b, ApJ, 572, L57

Lazzati, D. 2003, A\&A, 399, 913

McLaughlin, G. C., Wijers, R. A. M. J., Brown, G. E., \& Bethe, H. A. 2002, ApJ, 567, 454

Mészáros, P., \& Rees, M. J. 2001, ApJ, 556, L37

Paerels, F., Kuulkers, E., Heise, J., \& Liedahl, D. A. 2000, ApJ, 535, L25

Piro, L., Costa, E., Feroci, M., et al. 1999, ApJ, 514, L73

Piro, L., Garmire, G., Garcia, M., et al. 2000, Science, 290, 955

Rees, M. J., \& Mészáros, P. 2000, ApJ, 545, L73

Reeves, J. N., Watson, D., Osborne, J. P., et al. 2002, Nature, 416, 512

Reeves, J. N., Watson, D., Osborne, J. P., et al. 2003, A\&A, 403, 463

Ross, R. R., \& Fabian, A. C. 1993, MNRAS, 261, 74

Rutledge, S., \& Sako, M. 2003, MNRAS, 339, 600

Vietri, M., Ghisellini, G., Lazzati, D., Fiore, F., \& Stella, L. 2001, ApJ, 550, L43

Watson, D., Reeves, J. N., Osborne, J., et al. 2002, A\&A, 393, L1

Watson, D., Reeves, J. N., Hjorth, J., et al. 2003, ApJ, 595, L29

Woosley, S. E. 1993, ApJ, 405, 273

Woosley, S. E., \& Weaver, T. A. 1995, ApJS, 101, 181

Yoshida, A., Namiki, M., Yonetoku, D., et al. 2001, ApJ, 557, L27

Zycki, P. T., Krolik, J. H., Zdziarski, A. A., \& Kallman, T. R. 1994, ApJ, 437, 597 